\title{
Stress and Coping Strategies of Parents of Children with Special Needs
}

\author{
Windy T. de la Cruz \\ University of Negros Oriental-Recoletos \\ windycruz92@gmail.com \\ https://orcid.org/0000-0002-5890-6054
}

\begin{abstract}
In the Philippines, there is an increasing number of children with special needs, and parents' ability to cope has implications with the way they raise their child as well as in family relationships. This descriptive, comparative, and correlational study focused on determining the stress level of parents, their demographic characteristics, and coping strategies. Data were gathered using standardized instruments as well as profile sheets from the 57 parents who were chosen through the snowball sampling technique. Data were then analyzed using descriptive statistics, t-test, one-way ANOVA, and Pearson correlation coefficient. Findings showed a significant relationship between the stress level and educational attainment. Furthermore, parents utilized adaptive coping as their dominant coping strategy, but they were also at varying degrees and at different times of utilizing maladaptive coping strategies. The stress level was positively associated with maladaptive coping strategy suggesting that parents were prone to emotional problems. Results had implications for mental health practices in the country.
\end{abstract}

Keywords: Psychology, Stress, Coping Strategies, Descriptive-Correlational, Iloilo City, Philippines 